\title{
Evaluation and Commissioning of Commercial Monte Carlo Dose Algorithm for Air Cavity
}

\author{
Hideharu Miura ${ }^{1 *}$, Norihisa Masai ${ }^{1}$, Kouichi Yamada ${ }^{1}$, Junichi Sasaki ${ }^{1}$, Ryoong-Jin $\mathrm{Oh}^{1}$, \\ Hiroya Shiomi ${ }^{1}$, Muhammad Nauman Usmani ${ }^{2}$, Toshihiko Inoue ${ }^{1}$ \\ ${ }^{1}$ Miyakojima IGRT Clinic, Osaka, Japan \\ ${ }^{2}$ Department of Medical Physics \& Engineering, Osaka University Graduate School of Medicine, \\ Osaka, Japan \\ Email: *hide-miura@osaka-igrt.or.jp
}

Received December 3, 2013; revised January 2, 2014; accepted February 1, 2014

Copyright (C) 2014 Hideharu Miura et al. This is an open access article distributed under the Creative Commons Attribution License, which permits unrestricted use, distribution, and reproduction in any medium, provided the original work is properly cited. In accordance of the Creative Commons Attribution License all Copyrights (c) 2014 are reserved for SCIRP and the owner of the intellectual property Hideharu Miura et al. All Copyright (c) 2014 are guarded by law and by SCIRP as a guardian.

\begin{abstract}
The purpose of this study was to compare the Pencil Beam (PB) with Monte Carlo (MC) calculated dosimetric results using phantoms for air cavity region. Measurements in Tough water phantom with air gaps were used to verify the calculated dose. The plane-parallel ionization chamber was moved from $2 \mathrm{~mm}$ to $20 \mathrm{~mm}$ behind air gap. Calculations were performed for various air gaps $(1.0,2.0,3.0$ and $4.0 \mathrm{~cm})$ and field sizes $(4.2 \times 4.2,6.0 \times 6.0$ and $\left.9.8 \times 9.8 \mathrm{~cm}^{2}\right)$. The lateral missing tissue measurement was performed using the radiochromic RT-QA film. Dose difference between PB and chamber measurement near an air gap was greater for smaller field size, larger air gap thickness, and shallower depth behind air gap. As the distance from the phantom edge became shorter, the dose differences of the PB calculation and film measurement became larger. MC calculations were found within $3 \%$ agreement to the measured dose distributions. Our results demonstrate an excellent agreement between ionization chamber and radiochromic RT-QA film measurements and MC calculations.
\end{abstract}

\section{KEYWORDS}

Monte Carlo; Pencil Beam; Air Cavity; Treatment Planning

\section{Introduction}

The effect of heterogeneous corrections is an important issue that has increasingly drawn the attention of the medical physics community for last several years. In the report of Task Group No. 65 of the Radiation Therapy Committee of the American Association of Physicists in Medicine, inhomogeneity correction algorithms were categorized according to the level of anatomy sampled for scatter calculation and the inclusion or exclusion of electron transport [1]. The two photon dose algorithms available with the iPlan RT Dose (Brainlab, Munich, Germany) are the Pencil Beam (PB) and the Monte Carlo (MC) algorithms. Hurkmans et al. reported the limitations of dose calculations in the case of head \& neck tumor using the PB algorithm [2]. MC algorithm was able to predict the dose distributions with a higher accuracy

\footnotetext{
${ }^{*}$ Corresponding author.
}

[3]. Many researchers have investigated the effect of air cavities on the dose distribution and dose reduction near air cavity, depending on geometry, beam energy, and field size using various MC codes in water equivalent phantoms [4-7].

Fragoso et al. performed an experimental verification of the iPlan v. 4.1 MC algorithm, using water-, lung- and bone-equivalent materials to investigate the differences between measured and calculated dose distributions [8]. However, reports of PB and MC calculations for air cavity using commercial treatment planning system are lacking. The purpose of this study was the evaluation and commissioning of iPlan RT MC Dose algorithm for air cavity region. We compared the calculated dosimetric results between $\mathrm{PB}$ and $\mathrm{MC}$ algorithms using phantoms. In addition, we also compared the results of dose differences between $\mathrm{PB}$ and $\mathrm{MC}$ algorithms for maxillary cancer patient. 


\section{Materials and Methods}

Figure 1 shows the schematic of the experimental setup for dose measurements. Tough Water phantom of 3 and $20 \mathrm{~cm}$ thickness was used above and below the air gap, respectively. Source-to-surface distance (SSD) was set at $950 \mathrm{~mm}$. Tube voltage of $120 \mathrm{kV}$ was used in the computer tomography scans to produce a group of images of $512 \times 512$ pixels, with the slice thickness of $1.25 \mathrm{~mm}$. Markus parallel-plate ionization chamber (PTW, Freiburg) was used to measure depth dose distributions and dose profiles were measured using Gafchromic RTQA film. The plane-parallel ionization chamber was moved in $1 \mathrm{~mm}$ steps from $2 \mathrm{~mm}$ to $10 \mathrm{~mm}$ and in $2 \mathrm{~mm}$ steps from $10 \mathrm{~mm}$ to $20 \mathrm{~mm}$ behind the air gap. Central axis depth doses were measured with two different approaches. At first, the field size was kept constant to $4.2 \times 4.2$ $\mathrm{cm}^{2}$ and thickness of air gap was varied from $1.0 \mathrm{~cm}$ to $4.0 \mathrm{~cm}$ with the increments of $1.0 \mathrm{~cm}$. Then, the thickness of air gap was kept fixed to $3.0 \mathrm{~cm}$ for field sizes of $4.2 \times 4.2,6.0 \times 6.0$ and $9.8 \times 9.8 \mathrm{~cm}^{2}$.

The radiochromic RTQA (ISP Corp, Wayne NJ, USA) film was inserted into Tough Water phantoms using a constant field size of $9.8 \times 9.8 \mathrm{~cm}^{2}$. Epson Expression 1680 desktop flat-bed document scanner was used. Calibration curve was created in the following fashion: One sheet of film was cut into $3.0 \times 3.0 \mathrm{~cm}^{2}$ pieces and 6 pieces of film were irradiated to establish each calibration curve. Pieces of film were exposed to 50, 100, 150, 200, 250, 300 monitor units, respectively.

The plan was calculated with the iPlan RT Dose ver. 4.1.2 treatment planning system. Monitor units (MU) were determined from the prescribed dose to the isocenter, based on the PB algorithm. The plan was recalculated using MC algorithm while keeping the same planning parameters for beam arrangement, leaf positions, isocenter, position, and monitor unit, using the full MLC

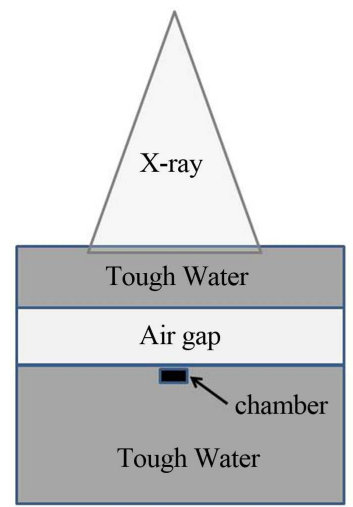

(a)

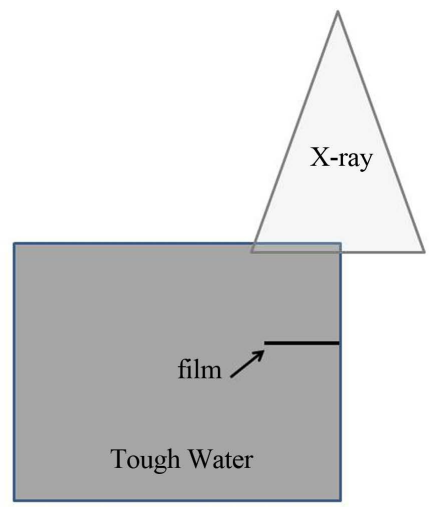

(b)
Figure 1. Schematic of the phantoms used in the chamber and film experiments. (a) The black box represents the plane-parallel ionization chamber; (b) The black line represents the RT-QA film. geometry simulation 'Accuracy Optimized Model' with a spatial resolution of $2 \mathrm{~mm}$ and variance of $1 \%$. The 6 MV photon beam energy was used from Novalis shaped beam radiosurgery unit (Brainlab, Munich, Germany). The charge for $200 \mathrm{MU}$ was measured three times for each position of the ionization chamber and the average of these measured values was used.

We selected the treatment plan of a patient who had been treated with Intensity-modulated radiation therapy (IMRT) for squamous cell carcinoma of maximally sinus. The gross tumor volume (GTV) was defined as the visualization of any gross disease. The clinical target volume (CTV) was defined as the GTV plus a $1 \mathrm{~mm}$ margin. The planning target volume (PTV) was defined as the CTV plus a $2 \mathrm{~mm}$ margin to account for tumor motion and setup uncertainty. GTV, CTV and PTV sizes were 1.5, 2.7 and 4.4 cc, respectively. A dose of $50 \mathrm{~Gy}$ in 10 fractions was prescribed to 95\% of the PTV (D95) with PB algorithm in this case. We recalculated the planned dose using MC algorithm while keeping the same number of MU per beam. A dose-volume histogram (DVH) analysis was performed for GTV, CTV and PTV. The brainstem, optic chiasm, eyes, and optic nerves were contoured as organs at risk (OARs). The following dose indices were used to evaluate the plan quality.

a) PTV D95; the minimum relative dose that covers $95 \%$ of the volume of the PTV.

b) PTV V95; the relative volume of PTV that receives at least $95 \%$ of the prescribed dose.

c) GTV D99 CTV D99; the minimum relative dose that covers $99 \%$ of the volume of the GTV and CTV, respectively.

\section{Results}

Figure 2 shows the dose distributions in a water phantom for $4.2 \times 4.2 \mathrm{~cm}^{2}$ field size with air gap. Significant dose difference can be observed between PB and MC calculations in re-build up region.

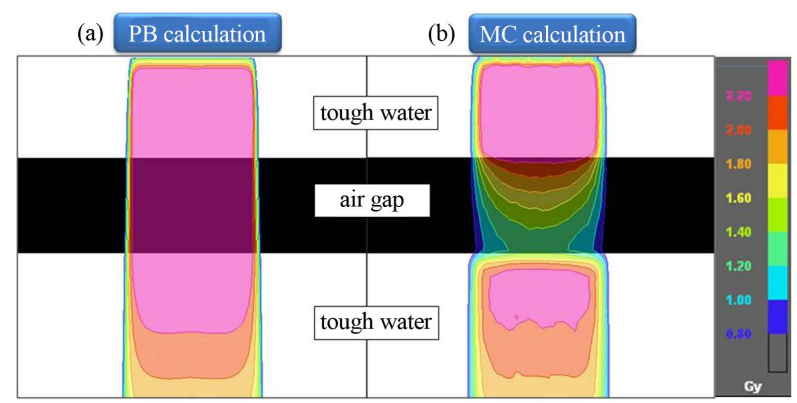

Figure 2. Dose distributions calculated using (a) PB and (b) MC algorithms in a Tough water phantom for $4.2 \times 4.2 \mathrm{~cm}^{2}$ field size with air gap. Compared to PB, MC calculations provide substantially lower doses to the air gap and rebuildup region. 
Figure 3 shows the calculated and measured data for various field sizes with a fixed air gap of $3 \mathrm{~cm}$. A direct relationship can be observed between calculated dose and field size with fixed air gap. PB calculated results did not match the measured data in re-build up regions. At $2 \mathrm{~mm}$ depth, the calculated results by the PB algorithm were $16.1 \%$ higher, whereas, MC calculated results were only $0.8 \%$ higher than measured dose with field size of $4.2 \times$ $4.2 \mathrm{~cm}^{2}$.

Figure 4 shows the calculated and measured data for various air gaps with a fixed field size of $4.2 \mathrm{~cm}$. An inverse relationship can be observed between calculated dose and air gap with fixed field size. PB calculated results did not match the measured data in re-build up regions. At $2 \mathrm{~mm}$ depth, the calculated results by the $\mathrm{PB}$ algorithm were $20.4 \%$ higher, whereas, MC calculated results were only 3.8\% higher than measured with air gap of $3.0 \mathrm{~cm}$.

Figure 5 shows the dose distributions calculated using $\mathrm{PB}$ and $\mathrm{MC}$ algorithms, and calculated and measured dose profiles in a water phantom for $9.8 \times 9.8 \mathrm{~cm}^{2}$ field size with lateral missing. Significant dose difference can be observed between PB and MC calculations near to the air gap. The $\mathrm{PB}$ results were $7.5 \%$ higher and $\mathrm{MC}$ results were $1.3 \%$ higher than measured dose at $10 \mathrm{~mm}$ from the phantom edge. As the distance from the phantom edge became shorter, the dose differences of the $\mathrm{PB}$ calculations became larger. The MC calculations and film measurements were in good agreement.

Figure 6 shows the comparison of dose distributions and DVHs for the PB and MC calculated plans. For PB calculated plan, the dose distribution was more homogeneous than MC calculated plan. The Table 1 compares the PB and MC calculated plans with regard to the PTV, CTV and GTV. D95 of the PTV, D99 of the GTV and D99 of the GTV using the MC algorithm were on average $26.4 \%$, 30.7\% and $25.8 \%$ lower than those with the $\mathrm{PB}$ algorithm, respectively. For OARs, the dose difference between PB and MC calculations is small.

\section{Discussions}

The results of the present study indicate the calculated dose differences between PB and MC algorithms. For both algorithms, the calculated dose increases with increasing field size. For MC algorithm, this effect is more significant in re-build up region showing a non-linear curve. For PB algorithm, this effect is comparatively less

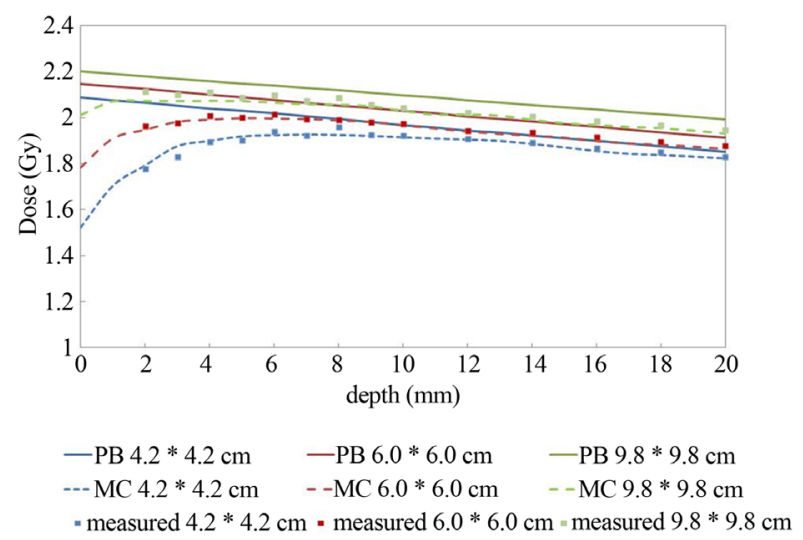

Figure 3. Depth dose in a Tough water phantom for $4.2 \times$ $4.2 \mathrm{~cm}^{2}, 6.0 \times 6.0 \mathrm{~cm}^{2}$, and $9.8 \times 9.8 \mathrm{~cm}^{2}$ field sizes with $3 \mathrm{~cm}$ air gap. Solid lines show the PB calculations. Dashed lines represent the MC calculations. Measured values are shown by dots.

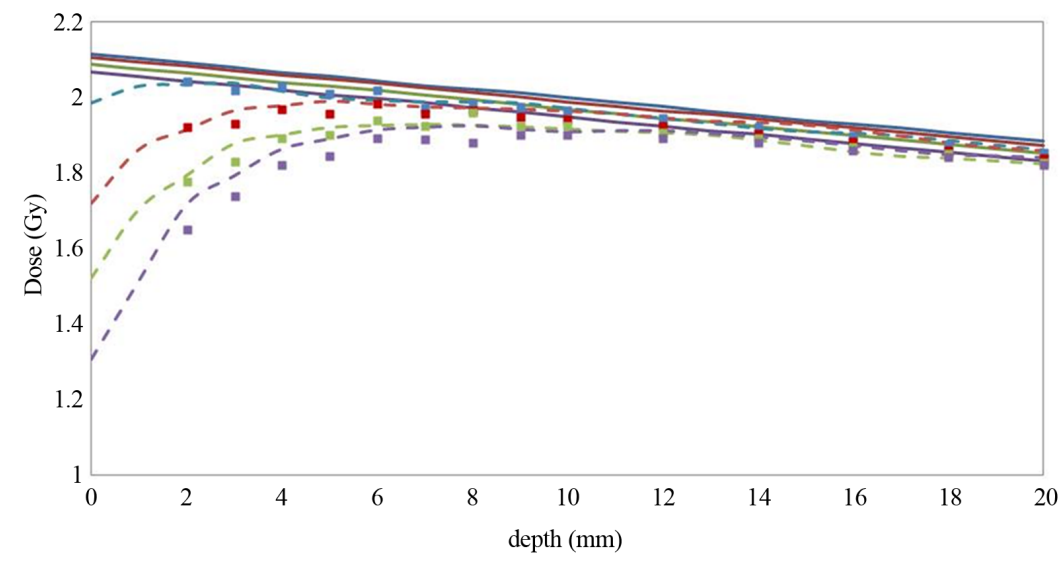

$$
\begin{aligned}
& \text { - PB air gap } 1.0 \mathrm{~cm} \quad \text { - } \mathrm{PB} \text { air gap } 2.0 \mathrm{~cm} \quad \text { - } \mathrm{PB} \text { air gap } 3.0 \mathrm{~cm} \quad \text { - } \mathrm{PB} \text { air gap } 4.0 \mathrm{~cm} \\
& \text { - - MC air gap } 1.0 \mathrm{~cm} \mathrm{-} \mathrm{-} \mathrm{MC} \mathrm{air} \mathrm{gap} 2.0 \mathrm{~cm} \quad \text { - - MC air gap } 3.0 \mathrm{~cm} \quad \text { - - MC air gap } 4.0 \mathrm{~cm} \\
& \text { - measured air gap } 1.0 \mathrm{~cm} \text { measured air gap } 2.0 \mathrm{~cm}=\text { measured air gap } 3.0 \mathrm{~cm} \text { " measured air gap } 4.0 \mathrm{~cm}
\end{aligned}
$$

Figure 4. Depth dose in a tough water phantom for $1-4 \mathrm{~cm}$ air cavities with $4.2 \times 4.2 \mathrm{~cm}^{2}$ field size. Solid lines show the PB calculations. Dashed lines represent the MC calculations. Measured values are shown by dots. 

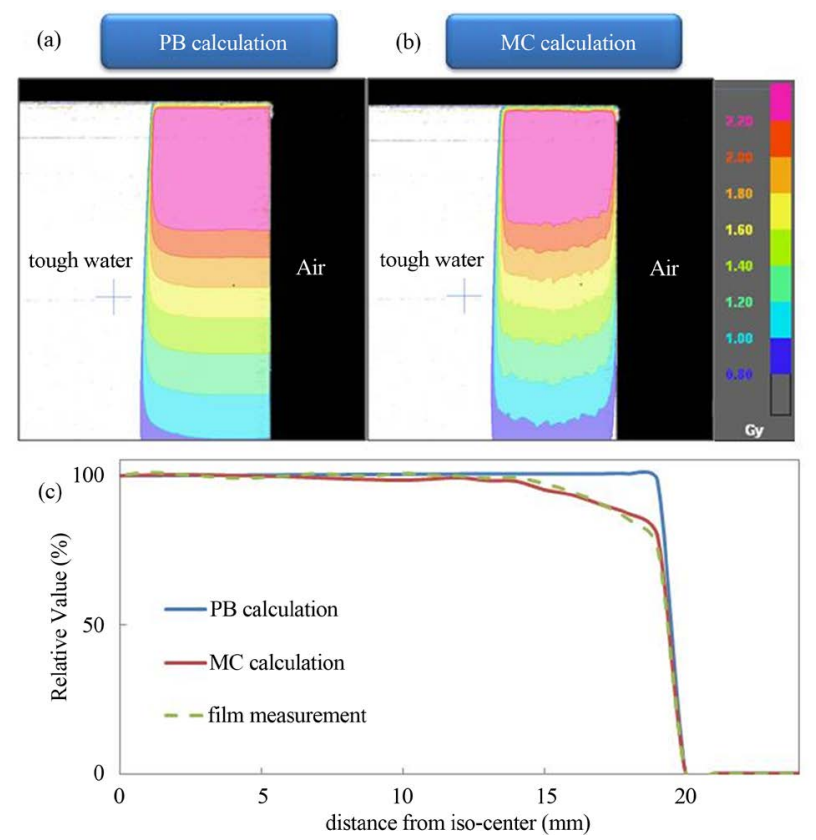

Figure 5. Dose distributions using (a) PB and (b) MC algorithms, and (c) calculated and measured dose profiles for the lateral missing phantom. Compared to PB, MC calculations provide substantially lower doses in the close proximity to the air.
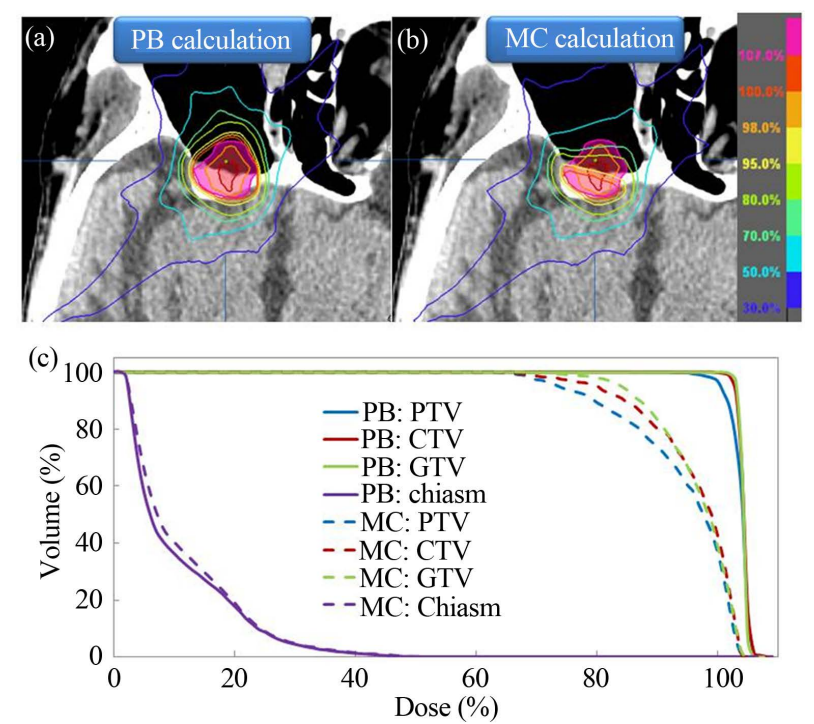

Figure 6. Planned dose distributions calculated by (a) PB and (b) MC algorithms for a patient with maximally sinus cancer; (c) comparison of dose volume histograms (DVHs) between PB (solid line) and MC (dashed line) calculated plans for the same patient. The PTV is shown as a translucent pink region.

prominent in re-build up region showing a linear curve. The PB calculated dose show a minor linear decrease in re-build up region with increase in the thickness of air gap. However, MC calculated dose reduces more significantly in re-build up region with increase in the size of
Table 1. Dosimetric comparison between PB and MC calculated plans.

\begin{tabular}{ccc}
\hline & PB (\%) & MC (\%) \\
\hline PTV & & \\
D95 & 100.0 & 73.6 \\
V95 & 99.7 & 60.4 \\
CTV & & \\
D99 & 100.1 & 69.3 \\
GTV & & \\
D99 & 102.2 & 75.9 \\
OAR: chiasm & & \\
D0.5cc & 1.52 & 1.7 \\
\hline
\end{tabular}

air gap showing a non-linear curve. The PB algorithms are able to account for the change of primary transmission in heterogeneous media with relatively simple algorithms, but cannot account for loss of electronic equilibrium at near tissue-air interfaces. MC algorithms model the actual physical processes leading to a dose deposition, including secondary electron distribution [9]. The reduction in dose to the points located beyond the air gap is due to a reduction in scattered radiation, produced in the material placed before the air gap. The loss in scatter contribution to the point of measurement is due to the lateral spread of the scattered radiation within the air gap. A re-build up region occurs as electrons are once again generated in water-equivalent material. Allen et al. reported that the dose reduction near an air cavity was greater for smaller field size, higher energy, larger air cavity size, and shallower depth in water where the air cavity was situated [10]. Klein et al. results showed that following a $2.0 \mathrm{~cm}$ wide air channel for a $4 \mathrm{MV}$ X-ray with $4 \times 4 \mathrm{~cm}^{2}$ field, there was an $11 \%$ under dose at the distal interface, while a $2.0 \mathrm{~cm}$ cubic cavity yielded only a 3\% loss [4]. Petoukhova et al. reported an excellent agreement of i Plan RT Dose MC calculation with the experimental data for phantoms with air cavities [11].

Behrens reported that the build-down effect was much smaller than the build-up effect and therefore not as important [12]. The dose reduction near an air cavity would have a negative clinical impact in a treatment if the region adjacent to the cavity consists of the target. Wang et al. employed the MC algorithm to assess the degree to which tissue inhomogeneities in the head \& neck affect static field conformal treatment plans. They concluded that the pencil-beam calculation corrected for primary attenuation by the equivalent path-length is a sufficiently accurate method for head \& neck treatment planning using 6-MV photons [13]. Yoon M et al. showed that when the beams pass through the oral cavity in anthropomorphic 
head \& neck phantom, the average dose difference becomes significant, revealing about $10 \%$ dose difference to prescribed dose at the isocenter [14]. Waldron et al. reported significant local recurrence rate of malignant disease in two separate retrospective trials involving 29 ethmoid sinus and 110 maxillary antrum cancer cases treated with curative intent. In these studies they expressed concern about the risk of local control of disease due to potential under dose of the target because of the physical uncertainties of the dose distribution achieved in irradiating large air cavities [15,16]. The limitation of this study was to use one beam irradiations. However, in a realistic therapy, a combination of multiple numbers of sub-fields within fields from multiple beam directions is used. Thus, the magnitude of interface dose reductions will likely be smaller than those for the limited number of radiation fields used in this study.

\section{Conclusion}

In conclusion, comparison of the depth dose and dose profiles between the measurements and MC calculations near air gap demonstrates excellent agreement concerning different air gaps and field sizes. We recommend that MC algorithm should be employed for accurate dose calculations in the presence of air cavity.

\section{Conflict of Interest}

None.

\section{REFERENCES}

[1] Task Group No. 65, the Radiation Therapy Committee of the American Association of Physicists in Medicine, “Tissue Inhomogeneity Corrections for Megavoltage Photon Beams. Madison,” Medical Physics Publishing, Madison, 2004.

[2] C. Hurkmans, T. Knöös, P. Nilsson, G. Svahn-Tapper and H. Danielsson, "Limitations of a Pencil Beam Approach to Photon Dose Calculations in the Head and Neck Region,” Radiotherapy \& Oncology, Vol. 37, No. 1, 1995, pp. $74-80$.

http://dx.doi.org/10.1016/0167-8140(95)01609-K

[3] E. K. Osei, J. Darko, A. Mosseri and J. Jezioranski, "EGSNRC Monte Carlo Study of the Effect of Photon Energy and Field Margin in Phantoms Simulating Small Lung Lesions,” Medical Physics, Vol. 30, No. 10, 2003, pp. 2706-2714. http://dx.doi.org/10.1118/1.1607551

[4] E. E. Klein, L. M. Chin, R. K. Rice and B. J. Mijnheer, "The Influence of Air Cavities on Interface Doses for Photon Beams," International Journal of Radiation Oncology*Biology*Physics, Vol. 27, No. 2, 1993, pp. 419-427.

[5] W. K. Kan, P. M. Wu, H. T. Leung, T. C. Lo, C. W. Chung, D. L. Kwong, et al., "The Effect of the Nasopharyngeal Air Cavity on X-Ray Interface Doses,” Physics in Medicine \& Biology, Vol. 43, No. 3, 1998, pp. 529-

\section{7. http://dx.doi.org/10.1088/0031-9155/43/3/005}

[6] B. H. Shahine , M. S. A. L. Al-Ghazi and E. El-Khatib, "Experimental Evaluation of Interface Doses in the Presence of Air Cavities Compared with Treatment Planning Algorithms,” Medical Physics, Vol. 26, No. 3, 1999, pp. 350-355. http://dx.doi.org/10.1118/1.598526

[7] I. Kawrakow, “Accurate Condensed History Monte Carlo Simulation of Electron Transport. I. EGSnrc, the New EGS4 Version,” Medical Physics, Vol. 27, No. 3, 2000, pp. 485-498. http://dx.doi.org/10.1118/1.598917

[8] M. Fragoso, N. Wen, S. Kumar, D. Liu, S. Ryu, B. Movsas, et al., "Dosimetric Verification and Clinical Evaluation of a New Commercially Available Monte Carlo-Based Dose Algorithm for Application in Stereotactic Body Radiation Therapy (SBRT) Treatment Planning," Physics in Medicine \& Biology, Vol. 55, No. 16, 2010, pp. 4445-4464. http://dx.doi.org/10.1088/0031-9155/55/16/S02

[9] M. Fippel, F. Haryanto, O. Dohm, F. Nüsslin and S. Kriesen, “A Virtual Photon Energy Fluence Model for Monte Carlo Dose Calculation," Medical Physics, Vol. 30, No. 3, 2003, pp. 301-311. http://dx.doi.org/10.1118/1.1543152

[10] X. A. Li, C. Yu and T. Holmes, “A Systematic Evaluation of Air Cavity Dose Perturbation in Megavoltage X-Ray Beams,” Medical Physics, Vol. 27, No. 5, 2000, pp. 10111017. http://dx.doi.org/10.1118/1.598966

[11] A. L. Petoukhova, K. van Wingerden, R. G. Wiggenraad, P. J. van de Vaart, J. van Egmond, E. M. Franken, et al., "Verification Measurements and Clinical Evaluation of the iPlan RT Monte Carlo Dose Algorithm for $6 \mathrm{MV}$ Photon Energy," Physics in Medicine \& Biology, Vol. 55, No. 16, 2010, pp. 4601-4614. http://dx.doi.org/10.1088/0031-9155/55/16/S13

[12] C. F. Behrens, "Dose Build-Up behind Air Cavities for Co-60, 4, 6 and 8 MV. Measurements and Monte Carlo Simulations," Physics in Medicine \& Biology, Vol. 51, No. 22, 2006, pp. 5937-5950.

http://dx.doi.org/10.1088/0031-9155/51/22/015

[13] L. Wang, E. Yorke and C. S. Chui, "Monte Carlo Evaluation of Tissue Inhomogeneity Effects in the Treatment of the Head and Neck," International Journal of Radiation Oncology*Biology*Physics, Vol. 50, No. 5, 2001, pp. 1339-1349.

[14] M. Yoon, D. H. Lee, D. Shin, S. B. Lee, S. Y. Park and K. H. Cho, "Accuracy of Inhomogeneity Correction Algorithm in Intensity-Modulated Radiotherapy of Head-andNeck Tumors,” Medical Dosimetry, Vol. 32, No. 1, 2007, pp. 44-51. http://dx.doi.org/10.1016/j.meddos.2006.11.004

[15] J. N. Waldron, B. O’Sullivan, P. Warde, P. Gullane, F. F. Lui, D. Payne, et al., "Ethmoid Sinus Cancer: TwentyNine Cases Managed with Primary Radiation Therapy,” International Journal of Radiation Oncology*Biology* Physics, Vol. 41, No. 2, 1998, pp. 361-369.

[16] J. N. Waldron, B. O’Sullivan, P. Gullane, I. J. Witterick, F. F. Liu, D. Payne, et al., "Carcinoma of the Maxillary Antrum: A Retrospective Analysis of 110 Cases,” Radiotherapy \& Oncology, Vol. 57, No. 2, 2000, pp. 167-173. http://dx.doi.org/10.1016/S0167-8140(00)00256-5 